\title{
A quality improvement project to improve the effectiveness and patient- centredness of management of people with mild-to-moderate kidney disease in primary care
}

\author{
Nicola Thomas, Hugh Gallagher, Neerja Jain \\ London South Bank University, Epsom and St Helier NHS Trust and Kidney Research UK
}

\begin{abstract}
Chronic kidney disease (CKD) stages 3 to 5 , affects $6-7 \%$ of the adult population and is an important risk factor for both advanced kidney disease and cardiovascular disease. This paper describes a quality improvement project that aimed to establish consistent implementation of best practice in people with stage 3-5 kidney disease who were managed in primary care.
\end{abstract}

The intervention was a Care Bundle for CKD. The bundle included three evidence-based, high impact interventions based on National Institute for Care Excellence (NICE, 2008) guidance, with an additional and novel self-management element.

29 GP Practices in England and Wales began the study. They undertook training in clinical management of CKD and in facilitation of selfmanagement, with the self-management content designed and led by patients. Practices were asked to report baseline and then monthly outcome data extracted from practice computer systems. The project team provided implementation and ongoing quality improvement support for participating Practices.

Ten Practices dropped out of the study following the training. Data submissions were incomplete in six Practices who continued to apply the care bundle. At the project end, a decision was taken by the study team to perform the final analysis on those thirteen Practices which completed the project and submitted at least six sets of monthly Practice-level outcome data. In these Practices the Care Bundle was applied to under $20 \%$ of the registered CKD stage 3 to 5 population in 5 Practices, $20-29 \%$ in 3 Practices, $30-49 \%$ in 2 Practices and $\geq 50 \%$ in 3 Practices (998 patients in total). Of these, 671 patients $(75 \%)$ agreed to the self-management component of the intervention.

The reliability (at project end) in those who received the Bundle was $100 \%$. The Bundle was applied to an additional 315 patients in the six Practices who completed the project but did not submit regular practice-level monthly data.

In the thirteen remaining Practices, the achievement of NICE (2008) blood pressure targets at the start of the project was $74.8 \%$ in people with CKD stage 3-5 and no diabetes and 48\% in people with CKD stage 3-5 and diabetes. At the project end these figures in the same Practices were $76.7 \%$ and $49.2 \%$ respectively. These improvements were achieved in spite of Practices increasing their recording of prevalence rate (that is, identifying and recording more patients with CKD on the Practice CKD Register).

In conclusion, a care bundle can be implemented in primary care. However, maintaining engagement with primary care health care professionals and maximising exposure to an intervention in patients seen infrequently are significant challenges to generalisation and sustainability.

\section{Problem}

There is widespread variation in the identification and treatment of kidney disease in primary care. The recorded prevalence of chronic kidney disease (CKD) within a Practice (measured by the number of patients on the CKD Register) is approximately $60 \%$ of that expected from epidemiological data (1). Performance against quality markers in those patients who have been correctly identified is also highly variable (1).

There is therefore a need for better systems to enable consistent implementation of best practice. However, the evidence base for quality improvement (QI) in mild-to-moderate CKD is limited. A systematic review identified very few studies involving $\mathrm{Q}$ I interventions to lower systolic blood pressure in CKD, and those there were largely focused on high risk groups (2), although more recent data suggest that a programme of audit-based education of healthcare professionals may be of benefit (3).

Furthermore, people with early CKD often wish to manage their condition with support from healthcare professionals (4), but anecdotal evidence from patients suggests that some healthcare professionals are not aware of or confident in encouraging their patients to self-manage. In addition, many people with a coded CKD diagnosis are not informed of it, and thereby denied any opportunity to self-care (5). Few CKD-specific educational materials have been developed to support those with early stage disease. 
We therefore designed and tested an intervention to: facilitate the delivery of consistent care to people with CKD in primary care; and offer patients the opportunity to self-manage.

\section{Background}

Chronic kidney disease stages 3 to 5 (CKD) affects between 6 and $7 \%$ of the population (6). It is an important risk factor for both endstage renal disease and for cardiovascular disease (CVD) $(7,8)$, with which it frequently co-exists, but is frequently unrecognised, since it causes no symptoms in the early stages.

In the UK, CKD is generally managed in primary care, and has been included under the General Practice pay-for-performance scheme since 2006. NICE guidelines on the identification and management of CKD were published in 2008 and will be updated in 2014 (9). Early identification is beneficial as there are effective interventions to slow progression and reduce cardiovascular risk (10). Implementation of best practice may be cost effective (11).

Best practice for CKD includes treatment of high blood pressure, with preferential use of drugs inhibiting the renin-angiotensin system where there is proteinuria. NICE also recommend that patients are asked about opportunities for self-management (9).

\section{Baseline Measurement}

In those thirteen Practices which completed the project and submitted at least six sets of monthly Practice-level outcome data the mean recorded prevalence of CKD at baseline was $4 \% \pm 1.54$ $\%$ (SD). The proportion of patients on the CKD Register treated to the NICE (2008) target for blood pressure control was $74.8 \%$ (with no diabetes) and $48.0 \%$ (with diabetes).

\section{Design}

We employed a Care Bundle approach. Care Bundles are groups of evidence-based, high impact interventions that have been well described in secondary care $(12,13)$. To our knowledge this is one of the first care bundle projects that have been described in primary care in England and Wales. A CKD-specific Care Bundle for primary care had been developed by the project team and piloted in a single Practice in 2008-2009 (14). Concurrently a patient-focused intervention had been designed by an expert group, which included patients. This too was piloted in a single Practice (15).

We combined the practitioner and patient components into a single Bundle, the components of which were:

- Asking the patient if they wished to participate in a selfmanagement programme

- Measuring and documenting proteinuria and prescribing an ACE inhibitor or Angiotensin Receptor Blocker (ACEi/ARB)

if significant proteinuria was present

- Documenting blood pressure and treating if above NICE target

- Documenting cardiovascular risk
The three clinical components of the Bundle are recommended by NICE (9). The self-management intervention, for those that chose to participate, involved offering an educational resource (DVD and written information) and short questionnaires/prompts to help patients identify their needs for discussion at their appointment.

Group education for patients

In addition a group education session for patients who had agreed to self-management was held in five Practices. Group education sessions were led by people who had CKD and were supported by a renal nurse. Sessions lasted two hours and included specific advice on how to self-manage early CKD (blood pressure control, tablets, diet, and smoking cessation) and also general advice on how to get the best out of a consultation with a doctor or nurse. People with CKD were therefore enabled to develop a pro-active interest in managing their health with a view to changing behaviour.

Training of Practices

Prior to commencing the project, participating Practices received dedicated training lasting 2-2.5 hours from two members of the project team (a renal nurse plus a member of the patient and service user advisory group (PSUAG), see below). The training covered both general information on CKD management in primary care and specific detail on the QI intervention to be implemented.

Patient involvement

The project was co-designed and delivered by patients. The PSUAG was established and its purpose was to help effect change and improvement in the involved Practices. This group consisted of six people who either had experience of kidney disease as service users/carers, or who had experienced other long-term vascular conditions (diabetes and heart disease). Their role was to develop and inform the project alongside other (clinical) members of the project team. Members of the PSUAG attended the grant application interview for project funding, created the selfmanagement package, led on the group education sessions for patients, and developed and delivered self-management training for practitioners (see below). They were supported by the wider team in these activities. Later in the project, PSUAG members played an important role in sustaining and disseminating the work, and contributed to follow-on project proposals.

Members of the PSUAG received the same honoraria for their time as healthcare practitioners in the project. The project team felt this to be an important factor recognising the value of service user involvement.

\section{Strategy}

Practices were asked to apply the Care Bundle to patients on their CKD Register. The project protocol did not prescribe a specific setting for application of the Bundle, but suggested settings included a dedicated CKD clinic, a generic long-term conditions clinic or ad-hoc delivery. 
Each month Practices were asked to send anonymised Care Bundle data to the project team. Each element of the Bundle was analysed separately as a process measure and overall Bundle reliability was recorded on a run chart. After collation of Bundle data, monthly teleconferences were held with participating Practices to discuss and review progress. The project team was represented on these calls by one of the project co-ordinators (a renal nurse) and, usually, a member of the PSUAG.

The Model for Improvement Plan-Do-Study-Act (PDSA) cycles were used as a framework. After each call the project co-ordinator emailed a summary of the call, tests of change, and action points to all those attending the call. At each subsequent teleconference the action points from the previous call were reviewed.

\section{Data collection}

Practice-level data outcome were collected at baseline, and then monthly, using data queries run on Practice computer systems. Data were sent electronically by Practices to the project team following email prompts from the project administrator. Search queries were written in-house by Practices, aided if necessary by the project team. Different IT systems were in use across the participating Practices.

The outcomes recorded were:

- Recorded prevalence of CKD (i.e. \% of adult population on CKD register)

- Proportion of patients on CKD Register treated to NICE target for blood pressure control (below 140/90 mmHg or below $130 / 80 \mathrm{mmHg}$ if patient had Type 1 or Type 2 diabetes and/or proteinuria (albumin-creatinine ratio, ACR > 70)

\section{Results}

We intended to achieve $95 \%$ reliability in the application of a Care Bundle for chronic kidney disease (CKD) in patients on the CKD Register within participating Practices by October 2012. Our main outcome goal was to achieve NICE targets for systolic blood pressure (BP) in $80 \%$ of people on the CKD Register within Practices (if $95 \%$ reliability not achieved).

Twenty nine GP Practices were recruited to the project and trained. Three Practices dropped out immediately after the training and three Practices never sent any baseline data, so twenty three Practices started the project. One Practice dropped out during the project. Thirteen of the remaining Practices sent at least 6 months practice-level data. An additional six Practices continued to apply the care bundle but sent less than 6 months practice-level data. These Practices have been excluded from our analyses. The Bundle was applied to an additional 315 patients in the six Practices who completed the project but did not submit regular practice-level monthly data.

Whilst we did not achieve our target, there were improvements in blood pressure management. In the 13 Practices who submitted $\geq 6$ months data, NICE blood pressure targets were achieved in $61.4 \%$ at the start of the project and $62.8 \%$ at the project end. These improvements were achieved in spite of a number of Practices increasing their recorded prevalence rate of CKD. One Practice increased their recorded prevalence rate by $3.5 \%$ ( 151 people) but at the same time increased the proportion of people achieving NICE blood pressure targets by $22 \%$. One Practice in North Wales saw its recorded prevalence rise from 4.8 to $7.6 \%$. Overall prevalence recording in the thirteen Practices increased during the project to $4.9 \%( \pm 1.62 \%)$

In thirteen Practices the Care Bundle was applied to $20-29 \%$ of the registered CKD stage 3 to 5 population in 3 Practices, $30-39 \%$ in 2 Practices and $\geq 50 \%$ in 3 Practices (998 patients in total). Of these, 671 patients $(75 \%)$ agreed to the self-management component of the intervention.

\section{Lessons and Limitations}

Intervention exposure

We demonstrated that the Care Bundle for CKD could be implemented reliably, and improvements in care were evident in some practices, such as increased prevalence recording and blood pressure control. The most challenging aspect of the project was achieving adequate intervention exposure. Our ability to assess the impact of the bundle upon practice-level measures has been limited by the proportions of patients on Practices' CKD Registers who received the intervention. A Practice with a list size of 10,000 may have 600-700 patients on their CKD register, a majority of whom might only be reviewed annually. Many of these patients have multiple co-morbidities and the settings for such reviews vary between Practices and between patients within Practices. The team therefore worked with a small number of Practices using a goaldefined approach to increase the intervention exposure, and by the end of the project more than $50 \%$ of registered patients had been seen in three Practices.

Achieving and maintaining 'buy-in' from Practices, especially in the ever-changing primary care environment, was also difficult.

Service-user involvement

The project's particular strength was the initiation and development of a patient and carer advisory group (PSUAG), that clearly demonstrated how patients and service users can meaningfully participate in quality improvement projects and lead on some elements.

Training

All Practices informally reported benefits of the initial training they received on CKD management. Even in Practices where the penetration of the Bundle was low, practitioners reported greater awareness of CKD and its treatment, and more tangible benefits in terms of improved identification, monitoring, and blood pressure management. One Practice reported that there had been a 'big impact of the education' on their already educated and relatively 


\section{BMJ Quality Improvement Reports}

affluent population.

Informing patients about their condition

In addition, practitioners reported that more patients were being advised of their diagnosis of CKD, which is widely recognised as an area of difficulty (16). Designing and delivering training was more complex than expected, as the motivations for participation in the project differed between Practices.

Impact

The impact of the Care Bundle varied across Practices and across areas of care within each Practice. Some Practices focused on the self-management aspects of the Bundle - in one practice over $90 \%$ of their patients agreed to take part in self-management - whereas others were less engaged in this element.

On the basis of our data it is not possible to distill the effects of the Bundle per se against the influence more generally within practices of a greater exposure to kidney disease. A number of factors outside the intervention and the project team will also have important impacts on performance, including pay for performance targets, Practice philosophy and the emphasis placed on education and decision-making, and nature of the consultation between individual health care professionals and the patient.

Participation

General practice as a setting for quality improvement projects presents unique challenges. Practices are small businesses and making the financial case for participation at the Practice level is important. Buy-in from a number of stakeholders was required for participation, typically a GP or Practice nurse "champion" within the Practice, GP partners, health care assistants, and practice managers. In some Practices the time taken from first expression of interest to delivery of the training programme was as long as four months. Even when the key people were enthusiastic, there was a significant amount of reinforcement and support required to embed the bundle. We also found that routine interruptions (e.g. Quality and Outcome Framework scheme pay for performance data returns, influenza vaccinations, staff changes, and sickness) distracted focus from the project.

Sustainability

A significant number of Practices did not complete the project. Stated reasons for this included a view that the 'project changed as it went on', the increased administrative burden of the extraction of evaluation data, a perceived need to recall patients earlier than would otherwise be necessary, issues around staffing in healthcare professionals and administrative staff, and the rapidly changing environment of the NHS/General Practice. However the differing levels of engagement across Practices does replicate that suggested by Rogers (17) and other studies of improvement science (18). Collecting monthly Practice-level data from Practices was particularly challenging and required a significant level of input and support from the project team. The team found that there was a wide range of capability amongst Practices with regard to their ability to manipulate the practice computer system.

\section{Benefits of Involvement to PSUAG team members}

The team was very focused on the meaningful involvement of patient and service user representatives through the PSUAG. An introductory paper, project overview, and role description were prepared by the chair of the PSUAG to ensure that group members had a common understanding of the project and its goals. The core project group placed great emphasis upon a flat team structure and inclusivity.

The PSUAG was involved in a number of crucial activities throughout the project. PSUAG members provided personal and anecdotal evidence about their own experiences which were used when talking to healthcare professionals who were considering joining the study and thereby supported recruitment. Patients and service users played a central role in the delivery of selfmanagement training and workshops to Practices and subsequent support at teleconferences with Practices. PSUAG members also developed and delivered the self-management training to patient groups.

\section{Conclusion}

We have demonstrated that a care bundle can be reliably implemented in primary care. However maximising exposure to the intervention in patients seen infrequently can be difficult, particularly outside the setting of a dedicated long term clinic. This in turn makes it difficult to evaluate the impact of the Bundle on care. The effect on biomedical outcomes at Practice level has been variable, and will have been influenced by factors both within and outside the study, such as the engagement of practice staff, the model for delivery of CKD care, and the Quality and Outcomes Framework cycle.

For a quality improvement intervention in primary care to be successful, it is important to have dedicated training on QI science prior to the start of the project, regular coaching from a QI team (from within or outside the organisation), service user (patient) involvement throughout, and buy-in from the senior management team such as GP partners and Practice managers.

We believe that a care bundle is a feasible vehicle for quality improvement in primary care, and that the lessons learned here may usefully inform further projects. Where there is a clear financial case for participation, test practices with a high degree of IT capability, and a patient population that is seen regularly in a defined setting, such an approach could facilitate the delivery of more consistent care. In kidney disease this might include a group at particularly high risk of adverse events (for example those with declining kidney function and heavy proteinuria, who can be identified using electronic searches) who are seen in a dedicated clinic. Any future Bundle might include a safety element as patients with CKD are at increased risk from poor prescribing. The involvement of patients on an equal footing from the outset will be a critical factor for success. 
Resources

All materials from the project can be found at

https://www.kidneyresearchuk.org/health-

information/resources/package-of-innovation

\section{References}

1. Health and Social Care Information Centre 2012; Quality and Outcomes Framework achievement data 2011/12 (http://www.qof.ic.nhs.uk/

2. Gallagher H, de Lusignan S, Harris K, Cates C. Qualityimprovement strategies for the management of hypertension in chronic kidney disease in primary care: a systematic review. British Journal of General Practice 2010; Jun;60(575):e258-65.

3. de Lusignan $\mathrm{S}$, Gallagher $\mathrm{H}$, Jones $\mathrm{S}$, Chan $\mathrm{T}$, van Vlymen J, Tahir A, et al. Audit-based education lowers systolic blood pressure in chronic kidney disease: the Quality Improvement in chronic kidney disease (QICKD) trial results. Kidney International 2010; 84:609-620

4. Constantini, $L$ et al The self-management experience of people with mild to moderate chronic kidney disease. Nephrology Nursing Journal 2008; Mar-Apr;35(2):147-55;

5. Health and Social Care Information Centre 2009. Health Survey for England, Volume 1: Health and Lifestyles (https://catalogue.ic.nhs.uk/publications/public-health/survey s/heal-surv-heal-life-eng-2009/heal-surv-heal-lifeeng-2009-rep-v2.pdf)

6. de Lusignan, S., Tomson, C.R., Harris, K., Van Vlymen, J., \& Gallagher, H. UK prevalence of chronic kidney disease for the adult population is $6.76 \%$ based on two creatinine readings. Nephron Clinical Practice 2012; 120, c107 7

7. Matsushita, K., van, d., V, Astor, B.C., Woodward, M., Levey, A.S., de Jong, P.E., Coresh, J., \& Gansevoort, R.T. Association of estimated glomerular filtration rate and albuminuria with all-cause and cardiovascular mortality in general population cohorts: a collaborative meta-analysis. Lancet 2010; 375, (9731) 2073-2081

8. Gansevoort, R.T., Matsushita, K., van, d., V, Astor, B.C., Woodward, M., Levey, A.S., de Jong, P.E., \& Coresh, J. 2011. Lower estimated GFR and higher albuminuria are associated with adverse kidney outcomes. A collaborative metaanalysis of general and high-risk population cohorts. Kidney Int; 80, (1) 93-104

9. National Institute for Health and Clinical Excellence. Early identification and management of chronic kidney disease in adults in primary and secondary care. NICE clinical guideline 73, 2008.

10. James MT, Hemmelgarn BR, Tonelli M. Early recognition and prevention of chronic kidney disease. Lancet 2010; 375(9722):1296-309.

11. Klebe B, Irving J, Stevens PE, O'Donoghue DJ, de Lusignan S, Cooley R, Hobbs H, Lamb EJ, John I, Middleton R, New J, Farmer CK. The cost of implementing UK guidelines for the management of chronic kidney disease. Nephrol Dial Transplant. 2007; 22(9):2504-12.
12. Resar R, Pronovost $P$, Haraden $C$, Simmonds $T$, Rainey $T$, Nolan $\mathrm{T}$. Using a bundle approach to improve ventilator care processes and reduce ventilator-associated pneumonia. Joint Commission Journal on Quality and Patient Safety 2005; 31(5):243-48.

13. Guerin K, Wagner J, Rains K, Bessesen M. Reduction in central line-associated bloodstream infections by implementation of a post-insertion care bundle. American Journal of Infection Control 2010; 38(6):430-33.

14. Thomas N. Can care bundles reduce variability in standards in primary care? British Journal Renal Medicine 2011; 16(1):19-22.

15. Thomas, N., Bryar, R. \& Makanjuola, D. Development of a self-management package for people with diabetes at risk of chronic kidney disease (CKD). Journal of Renal Care, 2008; 34(3), 151-8.

16. Abdi, Z., Gallagher, $\mathrm{H}$ and O'Donoghue, D. Telling the truth: why disclosure matters in chronic kidney disease. $\mathrm{Br} \mathrm{J}$ Gen Pract. Apr 2012; 62(597): 172-173.

17. Rogers E. Diffusion of Innovations. 4th ed. New York: Free Press, 2005.

18. Brandrud AS, Schreiner A, Hjortdahl P, Helljesen G, Nyen $B$, Nelson EC. Three success factors for continual improvement in healthcare: an analysis of the reports of improvement team members. BMJ Quality \& Safety 2011; 20(3):251-59.

\section{Declaration of interests}

HG is a member of the NICE Clinical Guideline Development Group (2012-2014) which is updating 2008 guidance on CKD. NT was a deputy on the NICE Clinical Guideline Development Group (2008).

\section{Acknowledgements}

All participating Practices and their patients, the Welsh Renal Network and the Primary Care Research Network.

Funding for the project was awarded by The Health Foundation under the 'Closing the Gap through Clinical Communities' award. 